http://jmscr.igmpublication.org/home/

ISSN (e)-2347-176x ISSN (p) 2455-0450

crossref DOI: https://dx.doi.org/10.18535/jmscr/v7i10.108

\author{
Dournal Of Medical Science And Clinical Research \\ IGM Publication \\ An official Publication of IGM Publication
}

\title{
Voice Outcome after Video Laryngoscopic Surgery on Benign Lesions of Vocal Cord
}

\author{
Authors \\ Ashna Sherief ${ }^{1}$, Shaiju $\mathbf{A}^{2^{*}}$, Satheesh $\mathbf{S}^{3}$ \\ ${ }^{1}$ Post Graduate Student, ENT Department, Govt. Medical College, Thiruvananthapuram \\ ${ }^{2}$ Associate Professor, ENT Department, Govt. Medical College, Thiruvananthapuram \\ ${ }^{3}$ Professor and HOD, ENT Department, Govt. Medical College, Thiruvananthapuram \\ *Corresponding Author \\ Shaiju A \\ Associate Professor, ENT Department, Govt. Medical College, Thiruvananthapuram, India
}

\begin{abstract}
Introduction: Benign lesions of vocal cord cause varying degrees of dysphonia and are surgically correctable. Microlaryngeal Surgery (MLS), voice rest and voice therapy offer cost effective, useful and safe method for management of these lesions ${ }^{1}$.

Objective: To evaluate voice improvement after videolaryngoscopic surgery in benign lesions of vocal cord and also to study the clinical profile.

Methods: Descriptive study conducted over period of 18 months. 50 patients with benign lesions undergoing videolaryngoscopic surgery studied. History elicited, examination performed, 70 degree endoscopy done, voice evaluated by VHI and GRBAS score, MPD and objectively evaluated by Praat software .Patients reassessed at 1,4 and 8 weeks after surgery.

Results: Male to female ratio was 4:1. Vocal cord polyps forms majority. Hoarseness present in all patients. Smoking and history of voice abuse forms major aetiological factor followed by acid reflux. The mean MPD pre-operatively was $7.08 \mathrm{sec}$ increased to $14.30 \mathrm{sec} 2$ months postoperatively. The mean VHI pre-operatively was 63.24 (severe) and mean VHI 2 months after surgery was 20.12 (normal) .Jitter became normal in 74\%, shimmer in only $34 \%$ of patients 2 months postoperatively.

Conclusion: Smoking, and voice abuse main aetiological factors. All the patients had normal vocal cord and near normal voice 2 months postoperatively. Subjective measures of voice outcome with GRBAS and VHI and also objective analysis with Praat showed signignificant improvement 2 months postoperatively. Jitter in Praat more relavant in monitoring response to treatment.

Keywords: Benign Lesions of Vocal Fold, Maximum Phonation Duration, Videolaryngoscopic Surgery.
\end{abstract}

\section{Introduction}

Hoarseness is defined as the perceived rough, harsh or breathy quality to the voice ${ }^{2}$. Normal voice requires laryngeal function to be coordinated, efficient and physiologically stable. Any imbalance in the delicate system can affect phonation $^{3}$. Benign lesions of the vocal folds can cause imbalances in this system which can result in varying degrees of dysphonia. Benign vocal fold lesions are broadly classified into neoplastic and non-neoplastic lesions. Benign non-neoplastic lesions are vocal nodules, vocal cysts, vocal polyps and papillomas. 
The most common etiology includes extroverts with a talkative personality and occupations with high voice demands. Other factors like smoking, acid reflux, uncontrolled allergy and infections are also involved.

With the advent of Videolaryngoscopic surgery it is possible to precisely remove lesions on the vocal cord without disrupting the delicate micro architecture of the vocal cord. Videolaryngoscopic surgery coupled with adequate voice therapy has been accepted as the standard treatment for benign lesions of vocal cord.

\section{Materials and Methods}

Descriptive type of study.50 patients with benign lesions of vocal cord (vocal cord polyp, vocal cord cyst, vocal nodule and papilloma) who are planned and worked up for Videolaryngoscopic surgery studied for a period of 18 months. Patients diagnosed as having benign vocal cord lesions on indirect laryngoscopy undergoing VLS included. Patients with inflammatory conditions like laryngitis, laryngeal tuberculosis, sulcus vocalis excluded.

Patients history is elicited and detailed systemic and ENT examination performed, 70 degree endoscopy examination done, voice of the patient is evaluated by VHI and GRBAS score, MPD and objectively evaluated by Praat software. Praat programme is a computer software programme ${ }^{4}$. The following valuables are recorded - jitter, shimmer, mean harmonics to noise ratio. Vocal nodules not responding to conservative management will be taken up for surgery. Patients will be reassessed at 1 week, 4 weeks and 8 weeks after surgery. At the time of follow up, they will undergo indirect laryngoscopic examination, 70 degree scopy and voice will be reassessed using the above measures.

\section{Results}

A total of 50 patients with benign lesions of vocal cord like vocal cord polyp, vocal cord cyst, vocal cord papilloma and vocal nodule who underwent videolaryngoscopic surgery were studied. There were 40 males (80\%) and 10 females (20\%) in the study. Male to Female ratio is 4:1.Majority of the patients were of the age group 41-50 years which was 18 in number accounting for $36 \%$ followed by $31-40$ years and 51-60 years each of $20 \%$. The youngest patient studied was 14 years and the eldest was 76 years.

Vocal cord polyps form the majority among the benign lesions of vocal cord. There were 40 cases of vocal cord polyp among 50 cases accounting for $80 \%$, there were 7 cases of vocal cord cysts (14\%), 2 cases of vocal cord papilloma (4\%) and only 1 case of vocal nodule (2\%). From this it is evident that majority of the vocal nodules regresses with voice therapy. Among the two cases of vocal cord papilloma one was a recurrence case.

Among 40 cases of vocal cord polyp there were 35 males and 5 females, among 7 cases of vocal cord cysts there were 4 males and 3 females, one case of vocal nodule was female. Even though males are more commonly affected the proportion of females with vocal cord cysts and nodule seem to be higher compared to polyps. In a study by Shaw et al incidence of vocal cord polyps was $71.2 \%$.

Regarding occupation $6 \%$ of patients were professional voice users (teacher, preacher and counsellor), majority were unskilled workers $(68 \%)$ followed by housewives (18\%), skilled workers $(6 \%)$ and student $(2 \%)$.
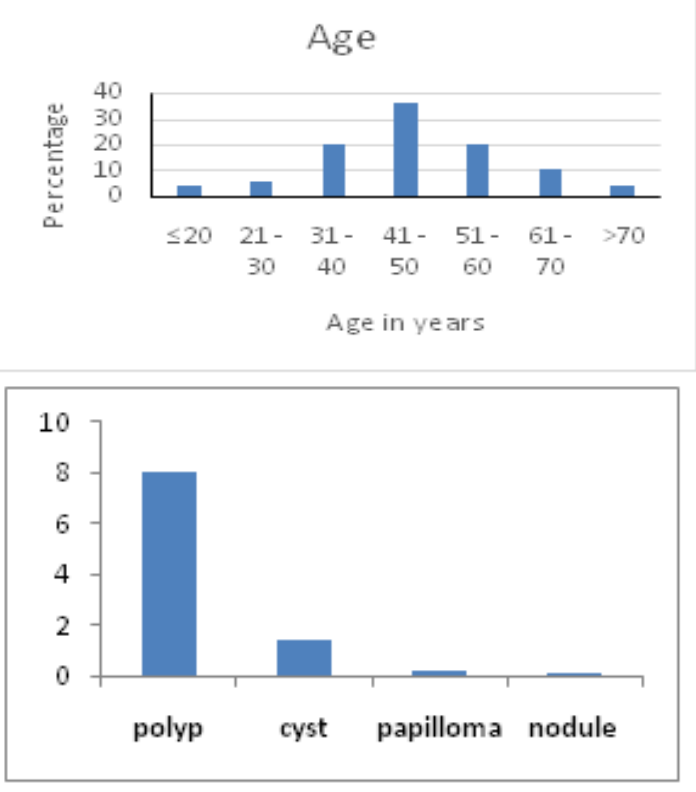
Hoarseness was the chief complaint in all the 50 patients studied (100\%). In addition to hoarseness 12 patients had voice fatigue $(24 \%), 6$ patients had foreign body sensation (12\%), 5 patients had difficulty in breathing (10\%), 4 patients had chronic cough (8\%) and 3 patients had hemoptysis $(6 \%)$.

In the study voice abuse and smoking form the main aetiological factors in causation of benign lesions of vocal cord accounting for $48 \%$ each followed by acid reflux in $28 \%$ cases.

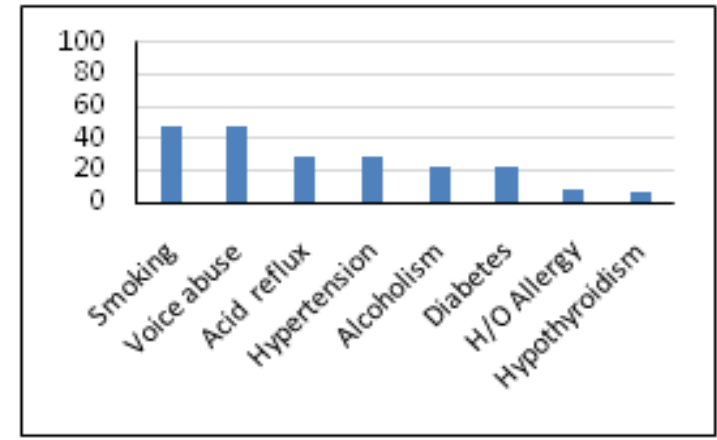

Indirect laryngoscopy was done pre-operatively, then by 1 week, 1 month and 2 months after surgery and findings analysed. Only 2 cases (4\%) showed normal vocal cords 1 week after surgery, others had congested vocal cords.46 cases(92\%) showed normal vocal cords 1 month after surgery, others had mildly congested vocal cords. All the cases $(100 \%)$ showed normal vocal cords 2 months after surgery.

Voice of the patients were evaluated preoperatively, 1 week, 1 month and 2 months after surgery. Voice evaluation done using VHI (Voice Handicap Index), MPD (Maximum Phonation Duration), GRBAS score and objectively evaluated using Praat software. In Praat software mainly jitter and shimmer values were assessed.

Pre operatively and 1 week after surgery none of the patients had normal MPD, $8 \%$ of patients had normal MPD 1 month after surgery. 2 months after surgery $62 \%$ of patients had normal MPD and $36 \%$ had subnormal values of MPD. This shows there has been a reasonable improvement in MPD. The mean MPD pre-operatively was $7.08 \mathrm{sec}$ which increased to $14.30 \mathrm{sec} 2$ months after surgery. In a study conducted by Nitish
Virmani and Arpit Sharma ${ }^{5}$ MPD increased from $9.43 \mathrm{sec}$ to $14.46 \mathrm{sec}$ at 6 months.

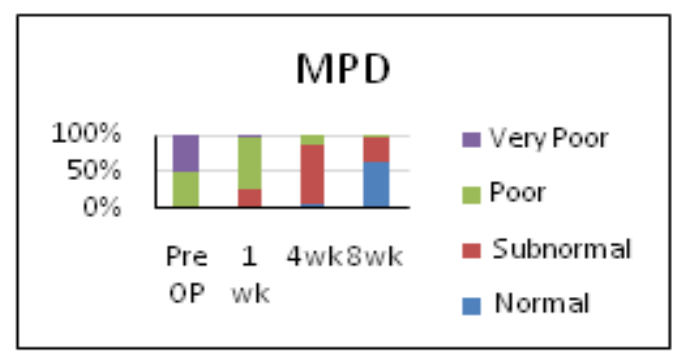

2 months after surgery the Grade of GRBAS score became normal in $62 \%$ of patients, mild impairment present in $34 \%$ of patients and moderate impairment in only $4 \%$ of patients. There has been a significant improvement in the Grade of GRBAS score.

Roughness of GRBAS score became normal in $46 \%$ of patients.

Breathiness of GRBAS score became normal in $68 \%$ of patients.

Asthenia of GRBAS score became normal in $86 \%$ of patients.

Strain of GRBAS score was normal in $86 \%$ of patients. Study by Nitish Virrmani and Arpit Sharma $^{5}$ the mean GRBAS score significantly improved from $7.5 \pm 0.82$ in the pretreatment stage to $2.3 \pm 1.17$ at 6 months

The mean VHI before surgery was 63.24 and mean VHI 2 months after surgery was 20.12. None of the patients had normal VHI pre operatively. 1 month after surgery $12 \%$ of patients had normal VHI and $86 \%$ had mild impairment. 2 months after surgery $62 \%$ had normal VHI and $36 \%$ of patients had mild impairment of VHI. In a study conducted by Ashwani Seth, Rakesh data in Army College of Medical Sciences the mean total pre-operative and post-operative VHI score were 61.69 and 10.04

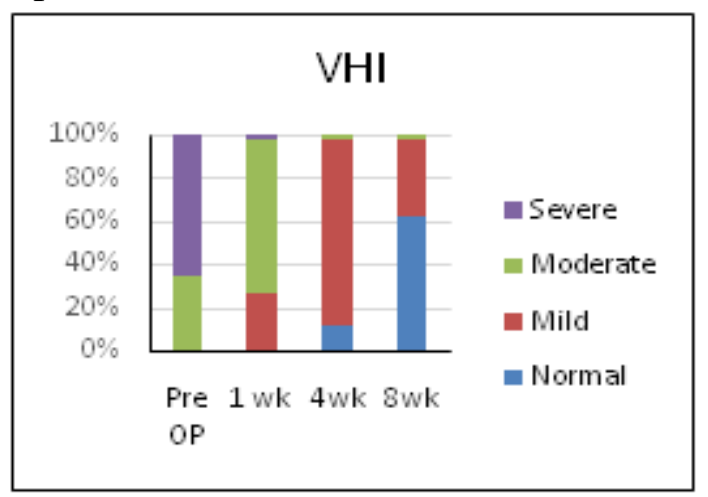


Voice evaluated using Praat software by measuring jitter and shimmer pre-operatively, 1 week, 1 month and 2 months after surgery. None of the patients had normal jitter value preoperatively. 1 week after surgery $8 \%$ had normal jitter value. 1 month after surgery $42 \%$ had normal jitter value and 2 months after surgery $74 \%$ of patients had normal jitter value. But shimmer value has come to normal in only $34 \%$ of patients which is low when compared to jitter. This shows jitter is most effective for monitoring treatment.

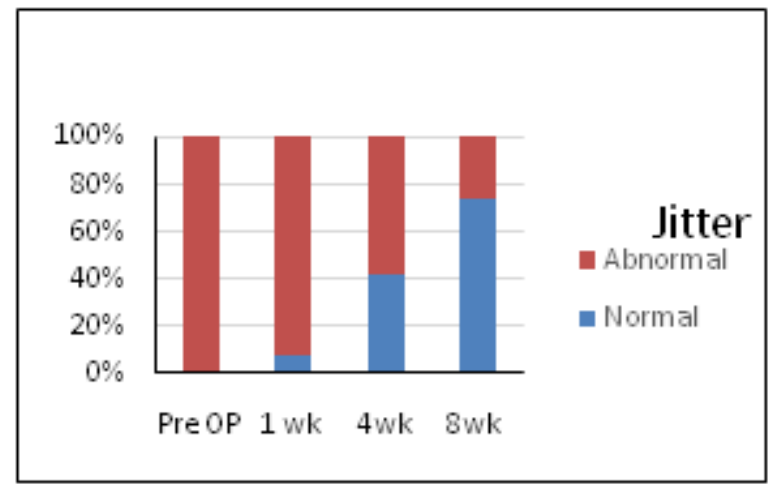

\section{Conclusion}

Vocal cord polyp forms majority among benign lesions of vocal cord accounting for $80 \%$ followed by vocal cord cysts (14\%), vocal cord papilloma $(4 \%)$, vocal nodule (2\%). Male to female ratio in the study was 4:1.Majority of patients were in the age group between $41-50$ years $(36 \%)$

Hoarseness was the commonest presenting complaint $(100 \%)$, followed by voice fatigue (24\%) and foreign body sensation (12\%).Smoking $(48 \%)$ and voice abuse (48\%) form the major aetiological factors in the causation of benign lesions of vocal cord, followed by acid reflux (28\%).

Indirect laryngoscopy showed complete clinical resolution of vocal cord pathology 2 months after surgery. On voice evaluation there has been significant improvement in the post-operative mean MPD value (7.08sec to $14.30 \mathrm{sec})$
There was significant improvement in the mean VHI score post-operatively (63.24 to 20.12). Perceptual voice scoring system GRBAS also show significant post-operative improvement in voice. In Praat software jitter value is more relevant compared to shimmer for monitoring treatment responses. Complete resolution of clinical and voice evaluation parameters occurs around 2 months postoperatively.

Videolaryngoscopic surgery offers a cost effective, useful and safe method for management of benign lesions of vocal cord

\section{Bibliography}

1. Titze IR Principles of voice production. Prentice Hall : Englewood Cliffs, New Jersey, 1994

2. Paul carding and Lesley Mathieson, Voice and speech production, Scott Brown's otorhinolaryngology, Head and neck surgery $7^{\text {th }}$ edition.

3. Steven Smith, Michael Underbrink, Benign Vocal Fold Lesions.

4. Boersma P. PRAAT, a system for doing phoenetics by computer GlotInt 2001 NovDec:5(9/10)341-345

5. Virmani N, Sharma A, Dabholkar J.Outcome Analysis in Patients with Benign Vocal Fold Lesions. Int $\mathrm{J}$ Phonosurg Laryngol 2016;6(1):8-13. 